DH Revista Humanismo

$R+10$ y Sociedad

\title{
Precisiones sobre el concepto de Imagen Corporativa ${ }^{\Perp}$
}

Clarification of the corporate Image Concept

Jaime Enrique Pallares Espinosa ${ }^{1^{*}}$, Comunicador Social Organizacional, Teólogo y Licenciado en Teología, Magíster en educación.

1 Docente en la Facultad de Comunicación Social-Periodismo de la Universidad Pontificia BolivarianaSeccional, Bucaramanga, de las asignaturas de Expresión Oral y Corporal, Ética, Deontología e Investigación, así como la Coordinación del Área de Comunicación Organizacional; Ha realizado investigaciones como: "Mapas Urbanos: una aproximación transdisciplinar al concepto de Agrupamientos"; "Observatorio Metropolitano de Comunicación Organizacional". Publicó el libro de investigación: "Impacto del Comunicador en Bucaramanga y su área Metropolitana". Líder del Grupo de Investigación en Comunicación Organizacional "Comité", registrado ante Colciencias.

(Recibido: 13 de mayo de 2014; aceptado: 24 de junio de 2014)

\section{Resumen}

La imagen corporativa es un componente fundamental en la proyección de las organizaciones hacia sus distintos grupos de interés. Diferentes agentes o profesionales intervienen en la presentación de la imagen corporativa, unos lo hacen con más propiedad y efectividad que otros. La investigación denominada "Caracterización del concepto de Imagen Corporativa que tienen las boutiques y los talleres de publicidad en Bucaramanga”, presenta un estudio comparativo sobre la comprensión que se tiene de dicho concepto en las entidades encargadas de asesorar a las empresas en sus elementos de imagen, pues si bien se trata de darle a las organizaciones una apariencia destacada que logre diferenciarlas en el Mercado global -según propósitos del megaproyecto Santander Visión 2030'-, proyección de la imagen no puede reducirse a un simple "maquillaje corporativo". Se consideró entonces pertinente realizar una investigación en dicho campo de la Comunicación Organizacional, en Bucaramanga, ya que se han identificado ofertas inadecuadas de diseño y mercadeo de la imagen corporativa.

Palabras clave: marketing, proyección de imagen, organizacional.

\footnotetext{
$\stackrel{\perp}{ }$ Para citar este artículo: Pallares Espinosa, JE. Precisiones sobre el concepto de Imagen Corporativa. RHS. Revista. Humanismo. Soc, Volumen 2 (1): 33-41.

*Autor para correspondencia: Jaime Enrique Pallares Espinosa. Universidad Pontificia Bolivariana - Seccional Bucaramanga. Correo: jaime. pallares@upb.edu.co

1 "Fomentar en el Departamento una cultura empresarial Global, direccionada a la creación de productos y servicios con alto valor agregado, que sean atractivos a los mercados internacionales. (Articulación con Santander Innovador y competitivo". En Macroproyecto Santander Global, Proyecto Santander Territorio de Negocios Globales. Tomado de:

http://www.uis.edu.co/webUIS/es/investigacionExtension/documentos/docInteres/Tomo\%20VI\%20Documento\%20\%20Proceso\%20\%20 Prospectivo.pdf consultado 12-06-2013
}

Revista. Humanismo.Soc. 2014; Volumen 2: 33-41. 


\begin{abstract}
Corporate image is a key component for projecting organizations towards their various stakeholders. Different agents and professionals are involved in presenting corporate image, with some doing it more properly and effectively than others. The study entitled "Characterization of the Corporate Image concept from boutiques and advertising workshops in Bucaramanga" presents a comparative study on how this concept is understood by the agencies responsible for advising companies on their image. Although it is aimed to stand out organizations in order to differentiate them in the global market -according to Santander Vision 2030 megaproject - the projected image should not be reduced to a simple "corporate makeup". A study was conducted in this field of Organizational Communication in Bucaramanga, where design and corporate image marketing offers have been identified.
\end{abstract}

Keywords: image projection, marketing, organizational.

\title{
Introducción
}

Uno de los elementos que aportan al "Top of mind" de una empresa es su identidad de marca, esto es, su logosímbolo o imagotipo; algunas compañías como la aerolínea colombiana Avianca se han atrevido a cambiarla a través del tiempo sin mayores repercusiones, otras como Coca-Cola han preferido no hacerlo para evitar confusiones en la recordación de sus clientes directos o potenciales. En relación con lo anterior, conviene sugerir que dicha identidad de marca hace parte del factor diferenciador de la compañía en el mercado global y si sufre muchas mutaciones, la consecuencia podría ser perderse en la jungla de marcas y logosímbolos del mercado internacional; si se tiene ya una imagen, un elemento gráfico bien posicionado en la mente del público, es mejor permanecer con ella como garantía. Así lo advierte el maestro Joan Costa cuando afirma:

"Respecto de la longevidad de los buenos nombres de marca, éste puede durar diez, veinte, cincuenta años o más. Algunos han superado el siglo de vida. El nombre es, pues, un instrumento estratégico potentísimo y de alta precisión que debe ser tomado con las mayores exigencias y precauciones" (Costa, 2004).

\section{Aporte teórico}

\section{¿Imagen corporativa o Identidad corporativa?}

Conviene despejar este interrogante porque se tiende a confundir elementos de imagen corporativa con identidad de marca y con elementos de identidad corporativa. Para evitar la confusión, es preciso distinguir los tres conceptos.
La Imagen Corporativa, en palabras de Nicholas Ind, "es la percepción que tiene un determinado público sobre una organización" (Ind, Nicholas-1992), comprende elementos tales como elementos visuales de la marca, slogan, publicidad, forma como la empresa es noticia en los medios, top of mind, reputación corporativa, atención al cliente y acciones de RSE. Un elemento visual o un slogan impactantes pueden ser arruinados por una pésima atención al cliente. Por otra parte, cuando se menciona Identidad corporativa se hace énfasis en la percepción que tiene la empresa sobre sí misma: "Incluye el historial de la organización, sus creencias, su filosofía, el tipo de tecnología que utiliza, sus propietarios, la gente que en ella trabaja, la personalidad de sus dirigentes, sus valores éticos y culturales, y sus estrategias" (Ind, Nicholas-1992).

Entre los elementos o signos de la identidad corporativa señalados en el documento titulado Diseño de Identidad Corporativa de la Universidad de Londres, cuyo compilador es el Licenciado Guillermo Albarrán Valenzuela, se encuentran el logotipo o imagotipo, la marca gráfica, el slogan, y los colores. El primero, es la versión gráfica del nombre de la marca, es decir, el tipo de letra; el slogan es la frase que va a llevar la empresa, por ejemplo la frase que identifica a la compañía Nike es "just do it”; la marca gráfica, corresponde a la parte icónica, distintivo figurativo, que siguiendo con el mismo ejemplo es el símbolo de Ok; y los colores son los tonos que van a distinguir a una empresa, que en este caso particular puede ser básicamente el negro y/o blanco, pasando por todos los colores de la paleta cromática, según el caso. 
Entonces, la Imagen Corporativa se percibe en el exterior mientras que la Identidad es percibida al interior de la organización; es decir, mientras que la identidad representa lo que es en sí la organización, la imagen corporativa, aclara Joan Costa, es la forma como conciben los consumidores esa determinada empresa, por ende los dos conceptos son complementarios pero totalmente opuestos. Conviene entender que la imagen corporativa es fundamental y determinante para el posicionamiento que tenga una empresa, ya que afianzará o no la percepción de sus clientes fijos o potenciales.

La identidad de marca está dada por el diseño gráfico de la misma. El experto en diseño gráfico Joan Costa indica:

Lo que llamamos el "lenguaje gráfico" es la combinación de tres modos fundamentales de lenguajes; ellos están ligados a la condición intrínsecamente comunicacional del grafismo y del diseño. La comunicación humana tiene su vehículo fundador en el Lenguaje, que es su modo específico de construir y transmitir significados. Pero 'los lenguajes' gráfico, gestual, cromático, etc. son extensiones visuales derivadas del Lenguaje por excelencia: el habla.

El lenguaje gráfico reúne y combina tres lenguajes básicos ligados a la Forma: son la Imagen, el Signo y el Esquema. Ellos corresponden 1) a la forma de las cosas del entorno, 2) a la forma de las notaciones y 3) a la forma de las estructuras que subyacen a las realidades invisibles (...) El cuarto lenguaje gráfico no es formal pero es subsidiario de la forma. Es el Color, pura sensación óptica que existe por la forma que lo contiene." (Costa, Joan-2004b)

Así pues, cada marca debe procurar diferenciarse de otra para afianzar su propia identidad corporativa, esto es, ser única, irrepetible, inconfundible. Sin embargo, podría contratarse a la misma Carolyn Davidson, estudiante de Diseño quien dibujó que el famoso logo de Nike denominado "Swoosh" (también conocido como "la pipa de Nike"), creado en 1971; pero si ocurre que a través de una investigación desarrollada por el canal 7 de Australia en 2008, el público se entera de que Nike realiza contratos irregulares y retiene personas de Indonesia y otros países asiáticos en las plantas de producción en Malasya, en condiciones infrahumanas, toda la inversión millonaria en publicidad, diseño gráfico, y el logro conseguido con el tiempo para que Nike sea número uno "Top of mind" de marcas, se va al suelo.

Para lograr el posicionamiento en el mercado global es preciso que tanto la identidad de marca como la Imagen Corporativa estén bien ubicas en el ranking mundial. Según el informe Global RepTrak 2012 Top 10 Brands, entre las cinco primeras marcas con mejor Reputación Corporativa mundial están BMW, SONY, Walt Disney Company, Daimler y Apple; sin embargo, dicha reputación ha sido ganada no sólo por un logosímbolo bonito, esto es, su identidad de marca sino por las prácticas coherentes entre lo que dice ser la compañía y lo que realmente hace, aspecto que le brinda la confianza de sus clientes y por ende hace fuerte su Imagen Corporativa.

En Colombia, y Bucaramanga, algunas empresas han pretendido mejorar la imagen de sus clientes cambiando elementos en su identidad de marca, en su slogan, en sus colores corporativos, en el empaque del producto, o realizando un despliegue de publicidad; sin embargo, ante las prácticas deshonestas en que caen, ante el incumplimiento de una promesa de venta, ante una mala atención al cliente, ante una denuncia o queja interpuesta ante la Confederación colombiana de consumidores, la imagen de la compañía queda afectada. Tal es el caso de la aerolínea Aires que fue comprada por la firma chilena LAN, mostrando cambios en su apariencia, pero incumpliendo en sus itinerarios y faltando a las promesas de servicio hechas a sus pasajeros; así lo evidencia el titular de Caracol Radio en su sitio web de 17 de enero de 2012: "Usuarios de LAN Colombia se quejan por fallas en compra de tiquetes por internet. Un usuario de la Aerolínea denunció que tras comprar su tiquete por Internet, cuando llegó al aeropuerto El Dorado, en la Aerolínea le afirmaron que no estaba registrado el pago"2.

Queda corroborado que la imagen corporativa no depende la identidad de marca o de algún elemento particular que la conforme, sino de la coherencia existente entre decir, hacer y cumplir. En palabras de Paul Capriotti, en el diplomado Comunicación 
Corporativa y Gestión de Crisis, módulo Gestión estratégica de la identidad y la imagen corporativa -Mendoza, Argentina, 2009-: "Gestionamos la identidad corporativa, gestionamos lo que nosotros somos, de hecho, es lo único que podemos gestionar. $\mathrm{Si}$ entendemos que la Imagen es lo que la gente piensa de la empresa, no se puede gestionar lo que la gente piensa de nosotros, porque hay muchas más cosas que tu punto de vista a la hora de formarse la opinión de los demás"; por tanto, no es posible gestionar, tener dominio sobre elementos de imagen corporativa, si bien la imagen es un valor estratégico de diferenciación que puede ser favorable o desfavorable.

Así pues, antes de pretender hacer "mejoras" en los elementos de imagen corporativa, conviene mejorar las prácticas de Atención al cliente. Miguel Antezana, experto latino en Comunicación Organizacional indica:

"La Imagen Corporativa es un asunto muy serio pues se trata de una responsabilidad compartida entre personas que -frecuentemente- no saben que tienen ese compromiso en sus manos. Siguiendo con el ejemplo, si las(os) vendedoras(es) de "Z" sólo tienen en mentevender la mayor cantidad de productos para ganar mayores comisiones y por ello convierten el establecimiento en un "campo de batalla", en el que los clientes son la presa a cazar, seguramente eso generará "ruidos" entre los visitantes al comercio. Estos "ruidos" pueden ser el casi acoso que sufre el posible comprador (al tener un vendedor "pegado" esperando alguna pregunta o preguntándole qué busca); o antagónicamente el poco interés de los vendedores; o la lentitud en responder a los requerimientos del cliente y una serie de posibilidades más que distan mucho del objetivo de "ser la zapatería con los mejores...”.

En realidad, estas situaciones son acciones comunicacionales (quizás involuntarias) de los trabajadores y que tienen como resultado "Imágenes" ante los públicos externos y que -con toda seguridadno son positivas y deterioran los mensajes que publicitariamente podría estar transmitiendo " $Z$ ". La Imagen Corporativa se genera con cada acción, pero también con la inacción de la empresa ante sus problemas ya sean internos o externos” (Antezana Miguel, 2009).
Lo anterior se refleja en la excelente atención al cliente que pueden mostrar en Colombia firmas como Porvenir fondo de pensiones y cesantías, cuyo certificado ISO 20000 y reconocimientos oficiales tales como Great Place to work, están soportados en los comentarios de satisfacción de sus afiliados; así lo evidencian su informe de gestión 2012 y la manera como la empresa es noticia en los medios ${ }^{3}$.

\section{Descripción de un "case"}

En Bucaramanga se encuentran boutiques y talleres de publicidad, que se encargan de diseño gráfico pero no está claro si se tiene en cuenta todos los aspectos comunicativos, a pesar de trabajar con los elementos de imagen corporativa de una empresa.

El registro mercantil de la Cámara de Comercio en 2010, en cuanto a empresas de publicidad señalaba en ese entonces la siguiente información:

\begin{tabular}{l|l} 
Empresas de publicidad & 301 \\
\hline Establecimiento de comercio & 290 \\
\hline Empleados & 552 \\
\hline Activos & $7,650,056,657$ \\
\hline Ventas netas & $13,711,324,615$ \\
\hline Patrimonio & $4,512,491,711$ \\
\hline Utilidad & $823,754,730$
\end{tabular}

Sin embargo, hay que tener en cuenta que en esta categoría no sólo se encuentran las boutiques y talleres publicitarios, sino que también incluye litografías, tipografías, empresas de estampado, talleres de diseño y demás.

Es claro, que en Bucaramanga son pocas las boutiques, se podrían señalar cuatro específicamente:

- Urbania

- Genoma Asociados Ltda.

- LCA Publiteca

- Pilonieta Vargas (PVS)

\footnotetext{
${ }^{3}$ Tomado de https://www.porvenir.com.co/NuestraCompania/Documents/PDF_info_responsabilidad_social_2012.pdf consultado 12-06-2013
} 
Las anteriores se categorizan como boutiques porque van en proceso de ser una agencia, es decir, son mini agencias que ofrecen un servicio más integral en la ciudad. Así mismo, cuentan con clientes de grandes empresas y tienen una experiencia en el mercado que las ha posicionado.

Por otra parte, existen aproximadamente 40 talleres de publicidad; algunos de estos son: 32 Bits, Agencia 55 pisos, AzuPublics, Bucaramanga en línea, Central Creativa, Concepto, Creativos, Eivos Ltda., Elemento Agencia De Publicidad, Galanés Publicidad, Hacemos Comunicación, Ideo Grupo, Inter Republic, Marketing U.P., Óptima comunicación, Opxion Publicidad y Mercadeo, Pauta Publicidad \& Comunicación, Portada Publicidad \& Marketing, Productiva, Publicity, Publingenio Creativos, Quántica, Taller Azul, Uno Publicidad, Wipe.

Además de los anteriores, también se ofrecen servicios de diseño de elementos de imagen, no sólo en sitios formalmente constituidos y matriculados comercialmente, sino en casas defamilia oapartamentos, así se evidencia en avisos mostrados en fachadas de casas en barrios residenciales que indican "Diseñamos su imagen corporativa". Lo anterior se constituye en un problema, pues si la imagen corporativa se reduce a sus elementos gráficos, cualquier persona con un computador puede hacerlo sin más requerimientos.

\section{Metodología}

Considerando los elementos anteriormente descritos, la estudiante Gloria María Martínez Salazar, especialista en Gerencia de la Comunicación Organizacional y egresada de la facultad de Comunicación Socialperiodismo, de la Universidad Pontificia Bolivariana, seccional Bucaramanga, realizó un ejercicio investigativo académico, asesorada por el profesor Jaime Enrique Pallares Espinosa, con el propósito de obtener su título como profesional; sin embargo, su graduación se dio por otra vía y cedió los derechos de la investigación a su tutor.

El objetivo general planteado fue caracterizar el uso del concepto de imagen de corporativa que tienen las boutiques y talleres de publicidad de Bucaramanga frente a las empresas; y como objetivos específicos describir los servicios que ofrecen las boutiques y talleres de publicidad en Bucaramanga, identificar el servicio que han solicitado las empresas que se han vinculado con las boutiques y los talleres de publicidad de Bucaramanga, y, señalar la percepción de las empresas sobre las boutiques y talleres de publicidad, y su concepto sobre la imagen corporativa.

El ejercicio investigativo fue de orientación metodológica exploratoria-descriptiva, enfoque mixto, que, según lo indica el investigador Roberto Hernández Sampieri "constituye el mayor nivel de integración entre los enfoques cualitativo y cuantitativo, donde ambos se combinan durante todo el proceso de investigación" (Hernández Roberto, 2004). Se utilizó este modelo porque no sólo se buscaba cuantificar la información sino también describir y analizar esos resultados. Con tal propósito, se estudiaron tres boutiques, ocho talleres publicitarios y sendas empresas que hubieran solicitado servicio de publicidad, aplicándoles las siguientes técnicas de recolección de información: la entrevista a profundidad, la encuesta y talleres de grupo. Con el fin de lograr el objetivo, se describieron los servicios que prestan estas boutiques y talleres de publicidad, se identificaron las empresas que han solicitado algún servicio publicitario y finalmente se indicó la percepción de éstas sobre el servicio recibido y el concepto que tienen acerca la imagen corporativa. En cuanto a lo cualitativo

El enfoque cualitativo "se basa en un esquema inductivo, es expansivo y por lo común no busca generar preguntas de investigación ni indagar hipótesis preconcebidas sino que éstas surgen durante el desarrollo del estudio, su método de análisis es interpretativo, contextual y etnográfico" (Hernández Roberto, 2004).

Este estudio es exploratorio porque en Bucaramanga no hay investigaciones sobre la concepción de imagen corporativa que tienen las empresas de publicidad, se indagó en un campo nuevo que aporta a los estudios de Comunicación Organizacional. Así mismo es descriptivo porque se trata de caracterizar y describir una situación; evalúa las características de una situación particular.

La aplicación de este método se dio por medio de la entrevista a profundidad. Se realizaron entrevistas a los directores de boutiques, de talleres de publicidad y a personas encargadas de mercadeo en las empresas que hayan contratado servicios publicitarios. Las entrevistas efectuadas a las personas en las boutiques 
y talleres se realizaron con el fin de cumplir con el objetivo general, y con el primer objetivo específico; por su parte, las entrevistas hechas a los representantes de las empresas que habían solicitado servicio publicitario respondieron al segundo y tercer objetivo específicos, basados en conocer los servicios que han solicitado y la percepción que tienen sobre las boutiques o talleres publicitarios.

La relación interpersonal conocida como el rapport (simpatía y concordancia entre el entrevistador y el entrevistado) facilita que se conozcan datos más profundos .Así mismo se adquiere más información porque no sólo se cuenta con la expresión oral sino también con los comportamientos del entrevistado.

Así mismo, se ejecutó un taller con los directores, ya fueran generales o creativos, de las boutiques y talleres de publicidad; por medio de éste se hizo un acercamiento al concepto que tienen de imagen corporativa.

\section{En cuanto a lo cuantitativo}

Según Roberto Hernández Sampieri (2004), este enfoque se fundamenta en un esquema deductivo y lógico, utiliza el análisis estadístico, es reduccionista y pretende generalizar los resultados de sus estudios a partir de muestras representativas.

De este enfoque, se realizaron encuestas a las empresas de Bucaramanga que habían solicitado algún tipo de servicio a una boutique o taller de publicidad, con el fin de conocer la percepción y la posición que tienen. Así mismo, identificar si se estaban satisfaciendo las necesidades del mercado santandereano.

"La encuesta como técnica de obtención de información, además de constituirse en un poderoso instrumento descriptivo resulta ser un útil instrumento explicativo" (Gaitán \& Piñuel, 1998), ya que del análisis sobre los resultados que arrojan la encuesta se obtienen conclusiones objetivas y subjetivas acerca del tema de estudio, es decir se encuentran descripciones y explicaciones.

Previamente se aplicó una prueba piloto de la encuesta, la semana del 12 al 18 de octubre de 2009, a un total de cinco empresas.

\section{Muestra}

La muestra fue no probabilística, es decir, que la población seleccionada no depende de la probabilidad sino de las características de la investigación. "La muestra no fue aleatoria sino que su selección se orienta en función de ciertos presupuestos teóricos $\mathrm{u}$ objetivos de la investigación" (Gaitán \& Piñuel, 1998).

Entre las boutiques de publicidad con las que se realizó el estudio se encuentran Genoma Ltda., Pilonieta Vargas (PVS), y LCA Publiteca. De los talleres de publicidad se seleccionaron ocho: Wipe, Galanés publicidad, Quántica, Óptima comunicación, Productiva, Bucaramanga en línea (BEL), Ideo Grupo y 32 Bits.

En cuanto a las empresas que habían solicitado servicios publicitarios se seleccionaron cuatro, de las cuales dos están vinculadas con alguna boutique de la muestra; una que solicitó servicios a un taller publicitario de la muestra; y una que estaba vinculada a una taller diferente a los de la muestra. Esto con el fin de tener una concepción más general.

Se seleccionaron las tres boutiques, los ocho talleres de publicidad y las empresas que solicitan servicios publicitarios de Bucaramanga que recibieron la invitación del estudio; por lo cual la muestra es representativa simbólica.

\section{Resultados}

\section{La encuesta}

Fue dirigida a 23 empresas de Bucaramanga que habían solicitado servicios en una empresa de publicidad. El instrumento se aplicó los días correspondientes a la semana del 19 al 31 de octubre de 2009. El objetivo de la encuesta fue conocer la perspectiva que tienen las empresas sobre las boutiques o talleres de publicidad donde han solicitado algún servicio; así mismo la concepción que tienen dichas empresa de la imagen corporativa. El modo de aplicación fue persona a persona y por internet.

El Formulario de la encuesta estuvo conformado por doce preguntas, y los resultados mostraron la siguiente tendencia: 
1. Las 23 empresas encuestadas respondieron que la publicidad sí es importante para una organización.

2. Catorce empresas han solicitado servicio de realización y ejecución de campaña publicitaria; cinco el servicio de creación de marca; tres el servicio de posicionamiento de la marca; y una servicio de investigación y estudio de mercadeo.

3. Ocho empresas han solicitado servicio en PVS; siete en otra agencia distinta a las que aparecen como opciones; seis en Genoma; y dos en Ideo Grupo.

4. De las siete organizaciones que habían solicitado servicio en otra empresa de publicidad respondieron: dos en Azupublics; dos en Urbania; dos en Wipe; y una en Futura.

5. Dieciséis calificaron con 5 el servicio brindado por la empresa de publicidad; seis con 4; y una con 3.

6. Ocho respondieron que NO hay nada por mejorar, continuar igual; cinco respondieron creatividad e innovación gráfica; cuatro respondieron eficiencia, tres respondieron mayor compromiso y contacto con el cliente; dos respondieron creatividad e innovación en el mensaje; una respondió diversidad de opciones para el cliente.

7. Doce respondieron que las empresas de publicidad existentes en la ciudad responden a las necesidades del mercado; 10 respondieron que algunas veces.

8. Ocho empresas respondieron que lo más importante de una pieza publicitaria es el diseño; seis respondieron el mensaje; cuatro respondieron la creatividad; cuatro respondieron la innovación; una respondió el nombre.

9. Once empresas respondieron que imagen corporativa son elementos gráficos y conceptuales; Seis respondieron identidad y organización de la empresa; Cinco respondieron proyección y visualización de la empresa; y una respondió lo que trasmite la empresa.

10. De acuerdo a la definición que dieron en la pregunta anterior, veintiún respondieron que las empresas de publicidad se encargan de la imagen corporativa de una organización; por su parte, dos respondieron No.

11. Doce empresas respondieron que imagen $\mathrm{e}$ identidad corporativa son lo mismo; once respondieron No.

12. Diecinueve respondieron que la presencia de la comunicación en la publicidad es muy necesaria; tres respondieron necesaria; y una casi innecesarios.

\section{Las entrevistas}

Después de realizar las entrevistas a personas a cargo de las once boutiques y talleres publicidad, se dedujo que: a pesar de tener claro las características propias de una agencia de publicidad, la mayoría considera que su empresa se encuentra en esta categoría. Señalaron la importancia de las investigaciones de mercado pero no es un servicio que ofrecen constantemente.

Sólo dos talleres publicitarios consideraron que la imagen es diferente a la identidad corporativa, y a pesar que muchos tienen dentro de su portafolio de servicios desarrollar la imagen corporativa, no tienen un concepto claro del mismo. Señalaron que muy pocas empresas se preocupan por su identidad e imagen corporativa, pues no comprenden la importancia de éstas. El problema de las empresas pequeñas es que no tienen mucho presupuesto para la publicidad, además de considerar esta herramienta de comunicación como un gasto y no como una inversión.

Consideraron importante la comunicación dentro de la publicidad, sin embargo vincular a un comunicador social a la boutique o al taller publicitario no es viable, ya sea por razones económicas o porque no es necesario, son pocas las que cuentan con este profesional en su equipo de trabajo.

En cuanto a las entrevistas hechas a los representantes de las cinco empresas que solicitaron servicios en dichas agencias: consideran que la publicidad es esencial, para dar a conocer la marca en el mercado y posicionarla en la mente de los consumidores y ante la competencia. Tres de ellas tienen un concepto acertado de Imagen Corporativa, sin embargo hay cierta confusión al tratar de diferenciarla con la Identidad Corporativa. 
Así mismo, afirman que las boutiques y los talleres de publicidad se encargan efectivamente de la Imagen Corporativa.

Consideraron que sí existen verdaderas agencias de publicidad en la ciudad, y que satisfacen sus necesidades. Algunos aspectos claves que deben tener en cuenta las empresas de publicidad: conocimiento de la empresa y del público objetivo, experiencia, calidad, confianza, cumplimiento, creatividad y originalidad.

\section{Análisis integrado de resultados}

Teniendo en cuenta los resultados de la encuesta, las entrevistas y las reflexiones realizadas con los equipos de trabajo de las boutiques y talleres de publicidad, se pudo hacer una triangulación de resultados, de cuyo análisis integrado se pudo deducir:

Las empresas de publicidad en Bucaramanga, no tienen un concepto acertado de Imagen Corporativa, por esto la tienden a confundir con la Identidad Corporativa. Los conceptos dados por las boutiques y talleres de publicidad no corresponden al de Imagen Corporativa.

De los resultados se infiere que aunque estas empresas ofrecen el servicio de Imagen Corporativa, no saben en realidad en qué consiste y orientan al cliente por otra perspectiva. No obstante, las empresas que contratan sus servicios afirman que se encargan efectivamente de la Imagen corporativa.

No sólo es una falencia de las boutiques y talleres publicitarios, sino también de las empresas que contratan sus servicios, pues la tienden a confundir con las características propias de la Identidad Corporativa o con aspectos meramente gráficos. No obstante, algunas empresas sí conocen el concepto pero consideran que las boutiques y talleres publicitarios lo manejan adecuadamente. Se infiere que hay poca documentación teórica y que sólo se limitan a los elementos de la imagen institucional (según el diseño gráfico).

Así mismo, siempre resaltan la importancia de la comunicación, pero la mayoría de las empresas de publicidad no consideran viable o no ven necesario la presencia de un comunicador social.
Las empresas de publicidad consideran quelas boutiques y talleres de publicidad ofrecen un buen servicio, basados en la calidad, la experiencia, la confianza, la originalidad, la creatividad y el conocimiento. Es decir, que aunque no son agencias de publicidad satisfacen el mercado de la región.

La publicidad es uno de los aspectos más importantes que debe tener en cuenta una empresa, pues es la forma de lograr un reconocimiento y de posicionar la marca. Sin embargo, en Bucaramanga se evidencia que pocas empresas invierten en publicidad.

\section{Conclusiones}

- Las empresas de publicidad en Bucaramanga, se clasifican de acuerdo a sus características en boutiques y talleres publicitarios, es decir no existen verdaderas agencias. Sin embargo, responden y satisfacen las necesidades del mercado.

- Las boutiques y los talleres de publicidad no aplican adecuadamente el concepto de Imagen Corporativa, y lo tienden a confundir con otros conceptos de la publicidad o del diseño gráfico. Esto demuestra, que no existe una revisión teórica de los conceptos que llegan a desarrollar.

- Las empresas conocen el concepto de Imagen Corporativa, sin embargo hay cierta confusión al tratar de diferenciarlo de la Identidad, lo que hace que lleguen a confundirlo.

- Las empresas consideran que las boutiques y los talleres de publicidad conocen y desarrollan bien el concepto de la Imagen Corporativa.

- La comunicación es esencial en la publicidad. Sin embargo, las boutiques y los talleres de publicidad no consideran necesario vincular a un comunicador social, lo que podría ser una falencia en el diseño de los mensajes de las diversas piezas o campañas publicitarias.

- Las empresas no solicitan el servicio de investigación de mercados, es decir, no conocen realmente el target al que desean dirigirse, ya que no conocen la opinión del consumidor antes de lanzar un producto o servicio al mercado. 
- Muchas empresas de la región consideran a la publicidad como un gasto y no como una inversión.

- Las empresas solicitan servicios publicitarios diariamente. Por esto, las boutiques y talleres de publicidad deben estar en constante evolución, ser innovadoras, generar confianza y conocer muy bien al cliente para poder satisfacer sus necesidades reales.
- La metodología fue apropiada porque se obtuvo la información necesaria para dar respuesta al objetivo del proyecto de investigación.

\section{Referencias}

Antezana, Miguel. (2009) ¿Sabes realmente de imagen corporativa? Revista dircom. Disponible en http://www. revistadircom.com/redaccion/comunicacion-corporativa/374isabes-realmente-de-imagen-corporativa-html

Costa, Joan. (2004) "Naming. Lo que no tiene nombre, no existe" http://www.reddircom.org/textos/naming.pdf

Costa, Joan. "Los tres fundamentos del Lenguaje gráfico". http://www.joancosta.com/docs/fundamentos.pdf

Gaitán Moya, Juan - Piñuel Raigada, José. (1998). Técnicas de investigación en comunicación social: Elaboración y registro de datos. España: Editorial Síntesis. Pág. 143
Hernández Sampieri, Roberto; Fernández Collados, Carlos; Baptista Lucio, Pilar. (2004) Metodología de la investigación. México, Ediciones Mc Graw Hill. Pág. 21.

Ind, Nicholas, (1992) La imagen corporativa: estrategias para desarrollar programas de identidad eficaces. Madrid: Ediciones Díaz de Santos. 